\title{
tRNA 3'-amino-tailing for stable amino acid attachment
}

\author{
HOWARD GAMPER and YA-MING HOU
}

Department of Biochemistry and Molecular Biology, Thomas Jefferson University, Philadelphia, Pennsylvania 19107, USA

\begin{abstract}
Amino acids are attached to the tRNA $3^{\prime}$-end as a prerequisite for entering the ribosome for protein synthesis. Amino acid attachment also gives tRNA access to nonribosomal cellular activities. However, the normal attachment is via an ester linkage between the carboxylic group of the amino acid and the $3^{\prime}$-hydroxyl of the terminal A76 ribose in tRNA. The instability of this ester linkage has severely hampered studies of aminoacyl-tRNAs. Although the use of $3^{\prime}$-amino-3'-deoxy A76 in a $3^{\prime}$ amino-tailed tRNA provides stable aminoacyl attachment via an amide linkage, there are multiple tailing protocols and the efficiency of each relative to the others is unknown. Here we compare five different tailing protocols in parallel, all dependent on the CCA-adding enzyme [CTP(ATP): tRNA nucleotidyl transferase; abbreviated as the CCA enzyme] to exchange the natural ribose with the modified one. We show that the most efficient protocol is achieved by the CCA-catalyzed pyrophosphorolysis removal of the natural A76 in equilibrium with the addition of the appropriate ATP analog to synthesize the modified 3 '-end. This protocol for $3^{\prime}$-amino-tailing affords quantitative and stable attachment of a broad range of amino acids to tRNA, indicating its general utility for studies of aminoacyl-tRNAs in both canonical and noncanonical activities.
\end{abstract}

Keywords: 3'-amino-3'-deoxy tRNA; CCA-adding enzyme; class I and class II; aminoacylation; prolyl-tRNA; streptavidinbiotin; aminoacyl-tRNA

\section{INTRODUCTION}

Amino acid attachment to tRNA generates the aminoacyltRNA (aa-tRNA) product that has wide-ranging activities in biology. In the canonical function, this attachment links an amino acid with the anticodon triplet of the genetic code and provides the basis to deliver the amino acid to protein synthesis on the ribosome at a codon position that matches with the anticodon. There are also noncanonical functions, however, in which an aa-tRNA is required for activity other than protein synthesis. Known examples of noncanonical functions include conversion of the glutamyl moiety of Glu-tRNA to Glu-1-semialdehyde for biosynthesis of porphyrin (Luer et al. 2005), utilization of the aminoacyl moiety of Tyr-, Leu-, and Phe-tRNA for biosynthesis of cyclic dipeptides (Gondry et al. 2018), and transfer of the aminoacyl group of Arg-tRNA to the $\mathrm{N}$ terminus of proteins to mark the arginylated proteins for degradation (Kashina 2014). Also well-known is the involvement of aa-tRNAs in biosynthesis of the bacterial cell envelope (Dare and Ibba 2012), which affects how the cell interacts with antibiotics and anti-microbial peptides. In both canonical and noncanonical functions, the aminoacyl linkage is the determinant of chemistry and is therefore of high interest to a broad range of research fields.

Corresponding author: ya-ming.hou@jefferson.edu

Article is online at http://www.rnajournal.org/cgi/doi/10.1261/rna. 068015.118 .
Amino acid attachment to tRNA is catalyzed in an ATPdependent reaction by the family of aminoacyl-tRNA synthetases (aaRSs) (Francklyn et al. 2002; Pang et al. 2014). The energy of ATP hydrolysis is used to activate the carboxyl group of the amino acid to esterify with one of the cis-diols of the terminal A76 ribose in tRNA. The site of aminoacylation is dependent on the aaRS. Due to their distinct structural and kinetic properties, aaRSs are divided into two classes (Eriani et al. 1990; Zhang et al. 2006), where class I enzymes aminoacylate the 2 '-hydroxyl, while class II enzymes aminoacylate the $3^{\prime}$-hydroxyl. After aminoacylation, the aminoacyl group migrates between the two diols in both directions without enzyme catalysis, although the 2'-to-3' direction is faster and is stabilized by interaction with the elongation factor $\mathrm{Tu}$ in bacteria (EF-1 in eukaryotes) for delivery to the ribosome (Taiji et al. 1983; Hansen et al. 2002; Zhang et al. 2006). However, the resulting ester linkage is unstable even at neutral $\mathrm{pH}$ (Peacock et al. 2014). This instability presents a challenge particularly in structural and biochemistry analyses, making it difficult to capture snapshots of the reaction chemistry in action. Historically, the solution to this instability problem

(C) 2018 Gamper and Hou This article is distributed exclusively by the RNA Society for the first 12 months after the full-issue publication date (see http://rnajournal.cshlp.org/site/misc/terms.xhtml). After 12 months, it is available under a Creative Commons License (Attribution-NonCommercial 4.0 International), as described at http:// creativecommons.org/licenses/by-nc/4.0/. 
has been to substitute the $3^{\prime}$-hydroxyl of the tRNA terminal A76 ribose with a $3^{\prime}$-amino group, which still permits aminoacylation by aaRSs but provides a stable amide linkage for amino acid attachment (Fraser and Rich 1973). We term this $3^{\prime}$-amino substitution of the tRNA terminal ribose a "tailing" procedure that establishes a molecular tail for stable attachment of amino acid.

A survey of the literature has identified multiple methods for tRNA 3'-amino-tailng, all of which use the CCA enzyme [CTP(ATP): tRNA nucleotidyl transferase] to catalyze exchange of the terminal A76 with the 3'-amino-3'-deoxy analog of ATP (abbreviated as nATP hereafter). CCA enzymes are present in all organisms and responsible for successive nucleotidyl addition to the tRNA $3^{\prime}$-end to synthesize and maintain the universally conserved $C^{74} C^{75} A^{76}$ sequence that encompasses the terminal A76 (Hou 2010). However, many of the published methods of 3'-aminotailing lacked a quantitative analysis of the yield and, even more problematically, none had been directly compared with others for efficiency, making it difficult to choose one method over another. Here we address this knowledge gap by parallel analysis of five different protocols, each from a published method, using the same tRNA substrate and CCA enzyme. To our surprise, we find that these five protocols vary widely in the efficiency of 3 '-amino-tailing, ranging from near complete conversion to as low as $1 \%-2 \%$. The most efficient method is the removal of A76 by pyrophosphorolysis of the CCA enzyme in equilibrium with the addition of nATP. Because inorganic pyrophosphate (PPi) is a reaction product of the CCA enzyme, the inclusion of PPi with the enzyme shifts the equilibrium backward, turning the enzyme into an exonuclease in a pyrophosphorolysis reaction that cleaves off A76. The presence of nATP in the equilibrium then facilitates the CCA enzyme to incorporate the analog to restore the tRNA to the full length. We show that the final product of this most efficient $3^{\prime}$-amino-tailing protocol is a tRNA that stably retains the aminoacyl group at the $3^{\prime}$-end generated by aminoacylation of either class of aaRSs. The applicability of the protocol to general aaRSs indicates its utility in studies of the tRNA aminoacyl group.

\section{RESULTS}

\section{Five protocols for tRNA $3^{\prime}$-amino-tailing}

We compared five protocols in parallel to determine the efficiency of each relative to the others (Table 1; Fig. 1). For this comparison, we used $E$. coli $(E c)$ tRNA ${ }^{\text {Pro/UGG }}$ (UGG: the anticodon) purified from native cells as the substrate, possessing all of the natural post-transcriptional modifications (Gamper et al. 2015). We chose Ec CCA enzyme to modify the tRNA $3^{\prime}$-end, based on our previous work showing that it is efficient in both the forward reaction of nucleotide addition and the reverse reaction of pyrophosphorolysis in the presence of PPi (Dupasquier et al. 2008; Kim et al. 2009; Igarashi et al. 2011). This CCA enzyme was purified to homogeneity from an over-expression clone (Hou 2000; Hou et al. 2005). In all five protocols, the CCA reaction was performed in the same buffer ( $50 \mathrm{mM}$ glycine, $\mathrm{pH} 9.0,10 \mathrm{mM} \mathrm{MgCl}$, and $1 \mathrm{mM}$ DTT) with the same concentration of the enzyme and tRNA (17 $\mu \mathrm{M}$ each). We maintained the enzyme at the tRNA substrate level to promote quantitative $3^{\prime}$-aminotailing.

TABLE 1. Protocols for $3^{\prime}$-amino-tailing of tRNA

\begin{tabular}{|c|c|c|c|c|c|}
\hline & \multicolumn{5}{|c|}{ Protocol } \\
\hline & 1 & 2 & 3 & 4 & 5 \\
\hline$E_{c}$ tRNA ${ }^{\text {Pro }}(\mu \mathrm{M})$ & 17 & 17 & 17 & 17 & 17 \\
\hline \multirow[t]{2}{*}{ Ec RNase T ( $\mu \mathrm{M})$} & 0 & 0 & 0 & 0 & 170 \\
\hline & - & - & - & - & $30 \mathrm{~min}, 37^{\circ} \mathrm{C}$ \\
\hline Ec CCA $(\mu \mathrm{M})$ & 17 & 17 & 17 & 17 & 17 \\
\hline \multirow[t]{2}{*}{$\mathrm{PPi}(\mathrm{mM})$} & 0 & 1 & 0.25 & 0.25 & 0 \\
\hline & - & - & $10 \mathrm{~min}, 37^{\circ} \mathrm{C}$ & - & - \\
\hline \multirow[t]{2}{*}{ CTP (mM) } & 0 & 0 & 10 & 0.62 & 0 \\
\hline & - & - & - & $10 \mathrm{~min}, 37^{\circ} \mathrm{C}$ & - \\
\hline PPiase (units) & 0 & 0 & 0.4 & 0.4 & 0 \\
\hline nATP (mM) & 1 & 6 & 2 & 2 & 1 \\
\hline Time (min) at $37^{\circ} \mathrm{C}$ & 30 & 60 & 30 & 30 & 30 \\
\hline
\end{tabular}

Ec CCA reaction buffer: $10 \mathrm{mM} \mathrm{MgCl}, 1 \mathrm{mM} \mathrm{DTT}$, and $50 \mathrm{mM}$ glycine, $\mathrm{pH} 9.0$.

Ec RNase T reaction buffer: $10 \mathrm{mM} \mathrm{MgCl} 2,50 \mathrm{mM} \mathrm{KCl}, 1 \mathrm{mM} \mathrm{DTT}$, and $20 \mathrm{mM}$ Tris- $\mathrm{HCl}, \mathrm{pH} 8.0$.

The incubation time after each addition is shown in bold.

In all protocols, the tRNA after the CCA reaction was phenol extracted, ethanol precipitated, and analyzed for $3^{\prime}$-amino-tailing. In protocol 5, an additional phenol extraction and ethanol precipitation was necessary after the RNase T reaction for 30 min. 


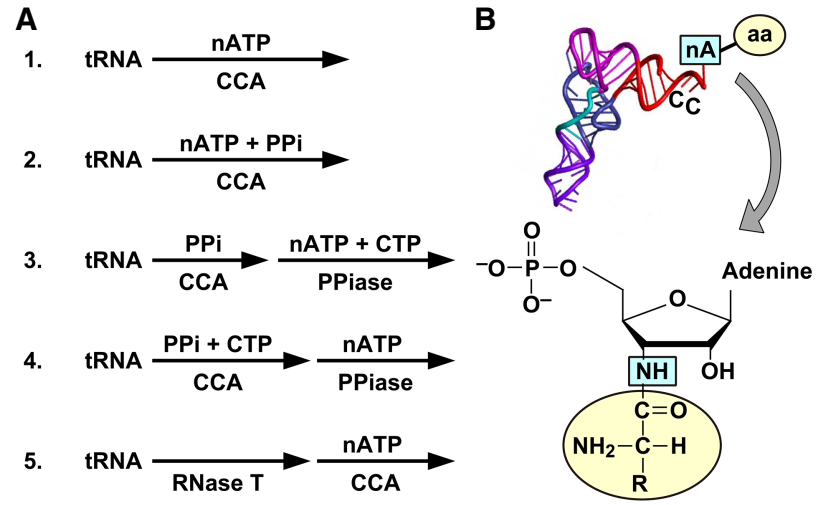

FIGURE 1. Five protocols of 3 '-amino-tailing of tRNA. (A) Protocols 15 are shown in the outlined format and each arrow indicates one reaction, where the enzyme is shown below the arrow and key reagents above the arrow. Of these, protocol 2 is the most efficient. (B) A tRNA L-shaped tertiary structure is shown, where the terminal nA nucleotide and the attached amino acid (aa) are shaded in light green and light yellow, respectively. The chemical structure of the ribose in $\mathrm{nA}$ is shown below, where the $3^{\prime}$-amino group is highlighted and its amide linkage to the amino acid is circled.

Protocol 1 was an exchange reaction typically performed to label the tRNA A76 with [ $\alpha-{ }^{32}$ P]-AMP (Peacock et al. 2014), but here the labeled nucleotide triphosphate was replaced with the modified nATP $(1 \mathrm{mM})$. Protocol 2 was also an exchange reaction, but it used PPi to activate pyrophosphorolysis to remove A76 while at the same time it performed incorporation of nATP to prevent pyrophosphorolysis of C75 or C74. The concentration of PPi and nATP (1 and $6 \mathrm{mM}$, respectively) and their ratio to each other was from a previous titration study (Francis et al. 1983). Both protocols 1 and 2 were performed in one step, allowing the forward and reverse reactions of the CCA enzyme to reach equilibrium.

Protocols 3-5 were each a nonequilibrium two-step reaction, where pyrophosphorolysis was performed ahead of nucleotide addition (Table 1; Fig. 1). Protocol 3 was initiated with the PPi-dependent pyrophosphorolysis to remove A76 in the first step, followed by hydrolysis of PPi with pyrophosphatase (PPiase) and addition of nATP for incorporation in the second step. CTP was also included in the nucleotide addition step to repair tRNA species missing C74 or C75 due to excessive pyrophosphorolysis. The concentrations of PPi, CTP, and nATP $(0.25,10.0$, and $2 \mathrm{mM}$, respectively) were from a previous method that prepared $3^{\prime}-{ }^{32} \mathrm{P}$-labeled tRNA for analysis of aminoacylation and peptide bond formation (Ledoux and Uhlenbeck 2008). Protocol 4 was also initiated with the PPi-dependent pyrophosphorolysis but in the presence of CTP to limit hydrolysis to A76. PPiase was then added in the second step to hydrolyze PPi while at the same time nATP was added for incorporation. The concentrations of PPi, CTP, and nATP $(0.25,0.62$, and $2 \mathrm{mM}$, respectively) were from a previous method that prepared tRNA with a 3'-amide linkage for X-ray crystal structural analysis of tRNA-ribosome complexes (Polikanov et al. 2014). Protocol 5 was unique in that, instead of pyrophosphorolysis, it removed A76 with RNase T, a bacteria-specific tRNA end-turnover enzyme that degrades the 3 '-end. This protocol was initiated in the first step with a 10-fold molar excess of RNase $T$ relative to $\mathrm{R}$ RA, which was determined necessary from a separate titration experiment. The tRNA was then phenol extracted and ethanol precipitated before its 3 '-end was extended with nATP in the second step by the CCA enzyme. The nATP concentration at $1 \mathrm{mM}$ of protocol 5 was similar to a previous method that used snake venom phosphodiesterase to remove A76 from tRNA (Fraser and Rich 1973). All five protocols were carried out at $37^{\circ} \mathrm{C}$, but with different time lengths based on the original conditions from which each was developed.

\section{Protocol 2 is the most efficient method for $3^{\prime}$-amino-tailing with nATP}

After reactions based on the five protocols in parallel, we analyzed the tRNA product of each for 3'-amino-tailing by biotin-streptavidin conjugation. Incorporation of the modified ribose of nATP in the 3 '-amino-tailed tRNA provides a unique primary amine to react with molar excesses of sulfo-NHS (N-hydroxysuccinimidyl)-D-biotin for conjugation with streptavidin. This tRNA-dependent biotinstreptavidin conjugation was previously used to monitor the presence of the primary amine after aminoacylation (Murakami et al. 2003). We subjected the tRNA product of each protocol to biotinylation, followed by conjugation with streptavidin. Because there was no other primary amine in each tRNA, the amount of biotin-streptavidin conjugation was a direct readout of the tailing reaction that would generate 3 '-amino-3'-deoxy ribose. The level of conjugation was quantified from a denaturing PAGE/ $7 \mathrm{M}$ urea gel, run at room temperature, which separated the untailed tRNA from the conjugate without dissociating the biotin-streptavidin complex from the conjugate (Fig. 2A). Due to the tetrameric nature of streptavidin, multiple 3'-amino-tailed tRNA molecules were captured, resulting in multiple biotinylated bands that up-shifted from the untailed band. By calculating the sum of all of the biotinylated molecules as the $3^{\prime}$-amino-tailed tRNA relative to the untailed one, we showed that the efficiency of tailing varied up to 80 -fold among the five protocols, reaching as high as $80 \%$ for protocol 2 and as low as $1 \%-8 \%$ for all others (Fig. 2B). This high degree of disparity between these protocols was previously unexpected.

As an independent analysis of 3 -amino-tailing, we determined the efficiency of the tailed tRNA for aminoacylation with ${ }^{3} \mathrm{H}$-proline, followed by alkali hydrolysis at $\mathrm{pH}$ 9.0. Aminoacylation was achieved with purified ProRS, a class II enzyme that aminoacylates directly the $3^{\prime}$-position of the terminal ribose. The presence of the $3^{\prime}$-amino-3'-deoxy 
A

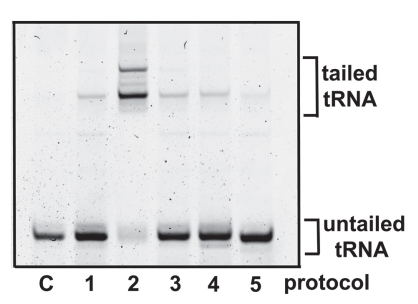

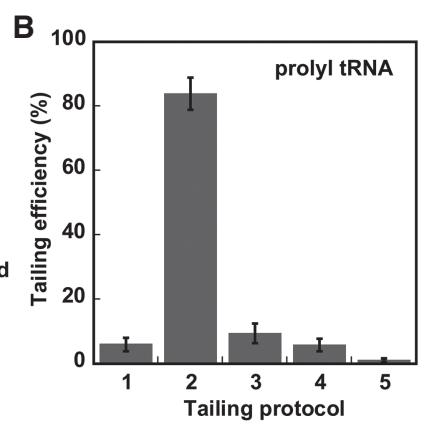

FIGURE 2. Analysis of $3^{\prime}$-amino-tailed tRNA by biotin-streptavidin conjugation. (A) Denaturing 12\% PAGE/7M urea gel analysis of conjugation on a Bio-Rad mini-gel apparatus, showing the up-shifted bands of tailed tRNA and the starting band of untailed tRNA. The gel was loaded with $\sim 70$ ng of tRNA in each lane and stained by SYBR gold. In the first lane, no upshifted band was observed with unreacted control (C) tRNA. (B) The tailing frequency of each protocol is calculated from the sum of all up-shifted bands over the amount of both shifted and unshifted bands. These data show that protocol 2 produces the highest level of 3 '-amino-tailing. Errors are standard deviation and $n=3$.

terminal ribose would capture the amino acid in an amide linkage, which is resistant to alkali hydrolysis. Thus, the level of ${ }^{3} \mathrm{H}$-proline that remained stably attached to the tRNA post-alkali hydrolysis, relative to pre-alkali hydrolysis, represented the efficiency of $3^{\prime}$-amino tailing (Fig. 3A). We showed that protocol 2 reached an efficiency of $90 \%$, whereas all other protocols reached no more than $8 \%$ (Fig. 3B), in agreement with the results determined by biotin-streptavidin conjugation (Fig. 2). Additionally, the efficiency of $3^{\prime}$-amino-tailing of each of the five protocols was quantitatively reproduced between the two detection methods, thus validating the level of 3 '-amino-tailing generated by each protocol.

\section{Stable aminoacyl attachment independent of the class of aminoacylation}

We determined if protocol 2 was similarly efficient with both classes of aminoacylation to produce high levels of stably charged amide-linked aa-tRNA. This was particularly relevant to class I aminoacylation, where the acyl group would be initially attached to the 2'-position of the terminal ribose in an ester linkage and then migrate to the $3^{\prime}$-position to form the amide linkage. While the transacylation (transesterification) migration is on the time scale of 2-10 $\mathrm{sec}^{-1}$ between the two hydroxyls in a normal ribose (Taiji et al. 1983), the rate of this migration in the modified ribose from the 2'-hydroxyl to the 3'-amino position had not been determined. Unless this rate was sufficiently fast within the timeframe of the experiment, protocol 2 would not capture the acyl group in the amide linkage. We tested the utility of protocol 2 by charging its product tRNA with enzyme-catalyzed aminoacylation for 10-15 min, followed by phenol extraction and ethanol precipitation, and by alkali hydrolysis at $\mathrm{pH}$ 9.0. We used ${ }^{3} \mathrm{H}$-amino acid for aminoacylation and determined the fraction of the label that remained stably charged to the tRNA after alkali hydrolysis. By analysis of tRNA ${ }^{\text {Arg }}$ and tRNA ${ }^{\text {Pro }}$ as the substrates for ArgRS (class I) and ProRS (class II), respectively, we found that the tRNA product of both classes, generated by protocol 2 , had the ability to stably retain the charged amino acid. In each case, while 3 '-amino-tailing compromised the charging capacity of the cognate enzyme (from $28 \%$ to $22 \%$ for tRNA ${ }^{\text {Arg }}$ and from $11 \%$ to $8 \%$ for tRNA ${ }^{\text {Pro }}$ ), it quantitatively stabilized the charged amino acid, whereas the untailed tRNA lost all of the charged amino acid after alkali hydrolysis (Fig. 4A). Notably, the quantitative retention of the charged amino acid, donated by class I ArgRS in aminoacylation, demonstrates that the transacylation of the aminoacyl group from the 2'- to the 3'-position of the terminal ribose had occurred before alkali hydrolysis.

To test more broadly the utility of protocol 2, we evaluated class I aminoacylation by MetRS and ValRS, in addition to ArgRS, and class II aminoacylation by LysRS, in addition to ProRS (Fig. 4B). The amino acid substrates among these enzymes differed in chemical structure, polarity, and ionic charges, and included the $\alpha$-imino group of Pro versus the $\alpha$-amino group of all others. The tRNA substrates among these enzymes differed in the primary sequence and the array of natural post-transcriptional modifications. In each case, the amino acid label that remained associated with the 3 '-amino-tailed tRNA post-alkali hydrolysis was measured and the level of retention

A
\begin{tabular}{|ccc|}
\hline \multirow{2}{*}{ Protocol } & \multicolumn{2}{l}{ Charged prolyl tRNA $(\%)$} \\
\cline { 2 - 3 } & $-\mathrm{pH} 9.0$ & $+\mathrm{pH} 9.0$ \\
\hline 1 & 8.5 & 0.66 \\
2 & 8.0 & 7.4 \\
3 & 8.2 & 0.65 \\
4 & 9.8 & 0.26 \\
5 & 7.6 & 0.041 \\
\hline
\end{tabular}

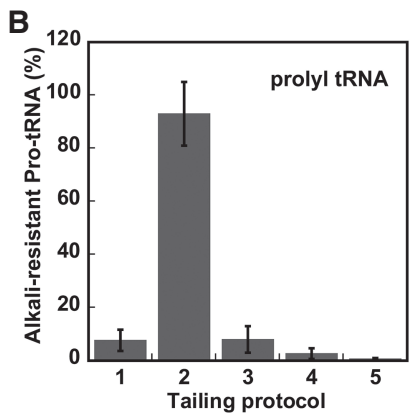

FIGURE 3. Analysis of $3^{\prime}$-amino-tailed tRNA by aminoacylation and alkali hydrolysis. (A) Levels of stably charged Pro-tRNA $A^{\text {Pro }}$ after alkali hydrolysis in $100 \mathrm{mM}$ glycine, $\mathrm{pH} 9.0$, for $3.5 \mathrm{~h}$ at $37^{\circ} \mathrm{C}$. In each protocol, the $3^{\prime}$-amino-tailed Ec tRNA ${ }^{\text {Pro }}$ was charged with ${ }^{3} \mathrm{H}$-Pro by the cognate ProRS, followed by alkali hydrolysis, ethanol precipitation, and measured for counts by a scintillation counter. The amount of label was measured before $(-)$ and after $(+)$ alkali hydrolysis and shown as the percentage of input tRNA Pro. For proline aminoacylation, charging by ProRS was usually in the range of $8 \%-9 \%$ as shown in the $-\mathrm{pH} 9.0$ column. (B) The level of alkali-resistant Pro-tRNA ${ }^{\text {Pro }}$ was determined from $A$ by measuring the ratio of post-alkali counts (+pH 9.0) over pre-alkali counts (-pH 9.0). These data show that protocol 2 produces the highest level of $3^{\prime}$-amino-tailing. Errors are standard deviation and $n=3$. 

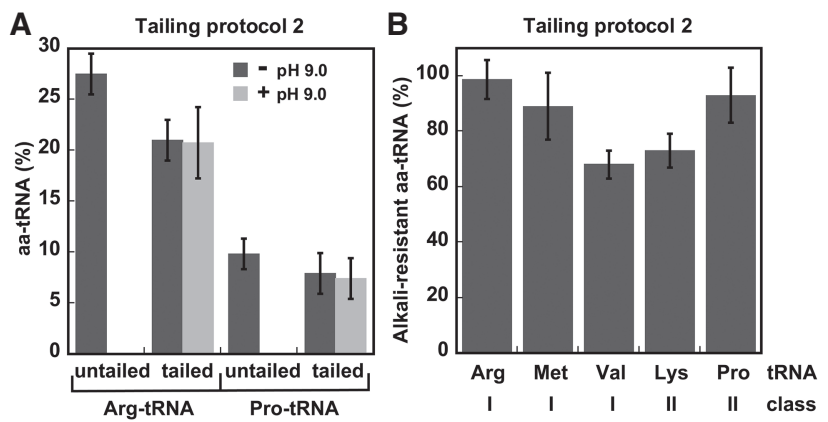

FIGURE 4. Stably charged amino acids attached to 3 '-amino-tailed tRNA generated by protocol 2. (A) Levels of Arg-tRNA and ProtRNA, with or without the tail, are measured pre- and post-alkali hydrolysis. Charging levels of untailed tRNA ${ }^{\text {Arg }}$ and tRNA ${ }^{\text {Pro }}$ are $28 \%$ and $11 \%$, respectively, whereas those of tailed tRNA $A^{\text {Arg }}$ and tRNA ${ }^{\text {Pro }}$ are $22 \%$ and $8 \%$. After alkali hydrolysis, levels of stably charged tRNA are measured. (B) Percent stably charged and alkali-resistant aa-tRNAs generated by class I ArgRS, MetRS, and ValRS vs. class II LysRS and ProRS aminoacylation of the cognate amino-tailed tRNAs. The lower levels of $\mathrm{Val}$ and Lys retention (70\%) relative to others $(90 \%-100 \%)$ were likely attributable to the quality of the starting tRNA samples rather than the identity of the amino acid. The methodology is described in Figure 3; errors are standard deviation and $n=3$.

relative to prehydrolysis was calculated. We showed that while the level of retention varied among different aminoacylation reactions, it was generally in the range of $70 \%-$ $90 \%$. For example, the level of retention for class I aminoacylation with $\operatorname{Arg}(100 \%)$, Met (90\%), and Val (70\%), was in the same range as that for class II aminoacylation with Lys (70\%) and Pro (90\%). There was no clear difference between the two classes, between the amino acids in each class, or between the tRNA substrates. We conclude that protocol 2 is applicable to both classes of aminoacylation to produce 3'-amino-tailed tRNA capable of generating high levels of amide-linked aa-tRNA.

\section{Time and nATP dependence of $\mathbf{3}^{\prime}$-amino tailing in protocol 2}

Protocol 2 was initially developed using yeast CCA enzyme to catalyze terminal nucleotide exchange in the presence of PPi and nATP for $1 \mathrm{~h}$. Because we used Ec CCA enzyme, we explored the time and nATP dependence of the exchange reaction. In a time course of the $3^{\prime}$-amino tailing reaction, analysis of the tailing level by the biotin-streptavidin conjugation method in denaturing gel (Fig. 5A) showed that the tailing progressed over time, starting from an initial time-dependent linear phase to reaching a plateau in 20 min (Fig. 5B). The stable plateau indicates the establishment of equilibrium between pyrophosphorolysis and nATP incorporation. The time parameters of nATP incorporation were closely similar to those previously determined for incorporation of 2'-dATP and 3'-dATP analogs (Francis et al. 1983), suggesting that protocol 2 has the capacity to incorporate a range of ATP analogs to study reactions at the tRNA $3^{\prime}$-end.

To determine the dependence on the nATP concentration for Ec CCA enzyme, we varied nATP between 0.5 and $6 \mathrm{mM}$. We found that, in the presence of $1 \mathrm{mM} \mathrm{PPi}$, the extent of $3^{\prime}$-amino tailing as shown in the gel mobility shift analysis reached the same high level (80\%) at all concentrations tested (Fig. 5C), whereas in the absence of PPi, the extent of tailing was low throughout. The finding that both the Ec and yeast CCA enzymes require $1 \mathrm{mM} \mathrm{PPi}$ for $3^{\prime}$-amino tailing indicates that they share in common the dependence on the initial pyrophosphorolysis reaction, but the finding that the Ec enzyme can reach high efficiency of tailing with only $0.5 \mathrm{mM}$ nATP is in contrast to that of the yeast enzyme, which requires $6 \mathrm{mM}$ nATP (Francis et al. 1983).
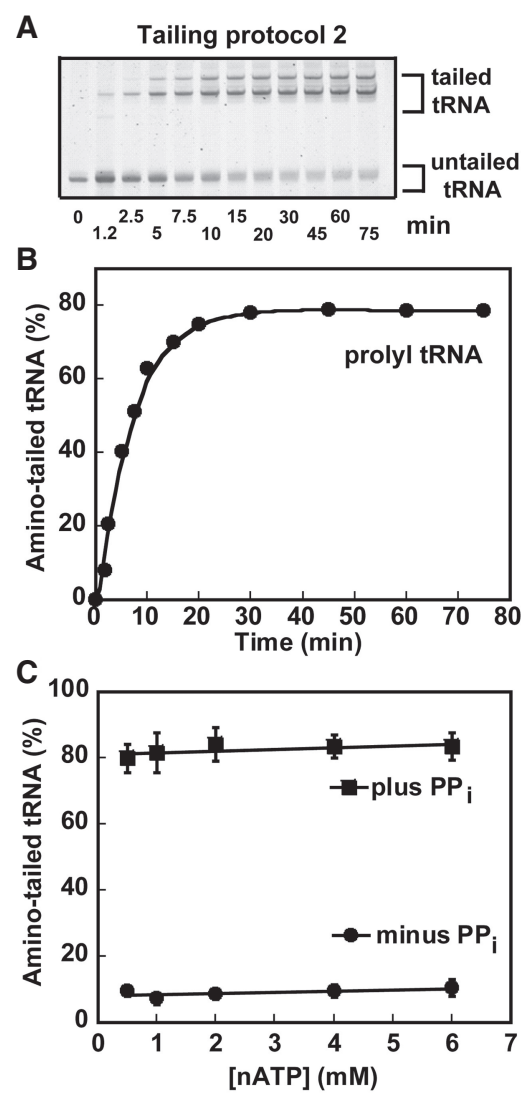

FIGURE 5. Time and nATP dependence of tRNA $3^{\prime}$-amino-tailing in protocol 2. (A) Denaturing 12\% PAGE/7 M urea gel analysis of 3 '-amino-tailing of Ec tRNA ${ }^{\text {Pro }}$ over time in protocol 2. After biotin-streptavidin conjugation, the tRNA (70 ng) was analyzed by denaturing PAGE. (B) The level of $3^{\prime}$-amino-tailed tRNA ${ }^{\text {Pro }}$ analyzed from $A$ is plotted over time, showing a plateau of tailing at 20-30 min. (C) Extent of $3^{\prime}$-amino-tailing of EC tRNA ${ }^{\text {Pro }}$ as a function of nATP concentration in the presence or absence of $1 \mathrm{mM}$ PPi. Reactions were incubated $60 \mathrm{~min}$ at $37^{\circ} \mathrm{C}$ using equimolar concentrations of tRNA and the CCA enzyme and analyzed as described above. Errors are standard deviation and $n=3$. 


\section{DISCUSSION}

Stable amino acid attachment to the tRNA 3 '-end via an amide linkage is a powerful and valuable mechanism to explore both canonical and noncanonical functions of the nucleic acid. The amide linkage is a close analog of the ester linkage, but it provides the stability needed to examine the structure of the aminoacyl group in action. While introduction of the terminal 3'-amino-3'-deoxy ribose moiety offered an attractive solution to enable a stable amide linkage, there had been multiple methods in the literature and their relative efficiency was unknown. Here, by parallel analysis of five protocols with the same tRNA substrate and CCA enzyme, we show that the most efficient is protocol 2, which performs equilibrium pyrophosphorolysis of A76 and incorporation of nATP. Based on our previous kinetic analysis of pyrophosphorolysis and nucleotide addition of Ec CCA enzyme (Dupasquier et al. 2008; Igarashi et al. 2011), we provide below a brief discussion for why protocol 2 outperforms the others.

Ec CCA enzyme catalyzes each forward and reverse reaction in a stepwise mechanism. The $K_{d}$ of nucleotide addition of $\mathrm{C74}, \mathrm{C75}$, and $\mathrm{A} 76$ is $\sim 40 \mu \mathrm{M}$, while the $K_{d}$ of PPi for pyrophosphorolysis from A76 to C75 to C74 is $\sim 0.5 \mathrm{mM}$ (Igarashi et al. 2011). In both forward and reverse reactions, the similar $K_{d}$ values for CTP- and ATP-dependent steps indicate that the enzyme can readily accommodate analogs of each nucleotide, such as nATP. Based on this assumption, protocol 2 is distinguished from the others by catalyzing the exchange of A76 in high concentrations of nATP $(0.5 \mathrm{mM})$ relative to the $K_{d}$ of nucleotide addition (40 $\mu \mathrm{M})$ and high concentrations of PPi $(1 \mathrm{mM})$ relative to the $K_{d}$ of nucleotide removal $(0.5 \mathrm{mM})$. These high concentrations serve to promote simultaneous pyrophosphorolysis of A76 and incorporation of nATP, consistent with the notion that both the forward and reverse reactions of CCA enzymes are concentration-dependent (Francis et al. 1983). By performing pyrophosphorolysis and nATP addition at the same time, protocol 2 is focused on the equilibrium exchange at the terminal position, without degradation of C75 and C74. In contrast, protocol 1 contains no added PPi and it relies on low endogenous levels of PPi to catalyze pyrophosphorolysis. This low level of A76 removal limits the extent of nATP addition and explains why protocol 1 is primarily used for $\alpha-{ }^{32} \mathrm{P}$-labeling of the terminal nucleotide, where radioactive substitution but not the quantity of conversion is of interest. Protocols 3 and 4 use PPi at a lower concentration $(0.25 \mathrm{mM})$ relative to the $K_{d}(0.5 \mathrm{mM})$ and perform nucleotide addition in a separate reaction. The independently run pyrophosphorolysis reactions open the possibility for removing $\mathrm{C} 75$ and $\mathrm{C} 74$, given the rate constant of pyrophosphorolysis $\left(k_{\mathrm{ppi}}=1-3 \mathrm{~s}^{-1}\right)$ and the timescale of the experiment (10 min, Table 1; Igarashi et al. 2011). Although CTP is included at a high concentration (10 $\mathrm{mM}$ ) during nucleotide addition in protocol 3, and at a low concentration $(0.62 \mathrm{mM})$ during pyrophosphorolysis in protocol 4 , the overall yield of both is poor. The mechanism for the poor yield is unknown, but may reflect that CTP inhibits pyrophosphorolysis and nucleotide exchange when starting from intact tRNA (Francis et al. 1983). Alternatively, the poor yield suggests that pyrophosphorolysis, when proceeding in the absence of equilibrium with nucleotide addition, is inefficient. Protocol 5 is distinct from all others by applying RNase $T$ to remove $A 76$ in one reaction and adding $n A T P$ in the next. This protocol consistently gives the lowest conversion yield, possibly due to a molar excess of RNase $T$ relative to tRNA resulting in hydrolysis of not only A76 but also C75 (Zuo and Deutscher 2002).

Protocol 2 generates high yields of 3 '-amino-tailed tRNA (70\%-100\%, Figs. 2, 3) and enables stable amino acid attachment (>70\%, Fig. 4). Protocol 2 has high efficiency with five different tRNA species that differ in sequence and post-transcriptional modifications and are substrates for either class I or class II aaRSs (Fig. 4). Although we only used Ec CCA enzyme to evaluate the various protocols, the quantitative yield of 3 '-amino-tailing in our protocol 2 is similar to that reported with the yeast enzyme (Francis et al. 1983). Notably, while the Ec and yeast CCA enzymes are structurally related (Yue et al. 1998), we find that they differ in the affinity for nATP in that while lower concentration of nATP $(0.5 \mathrm{mM})$ can saturate the Ec enzyme, higher concentrations of nATP $(6 \mathrm{mM})$ are required to saturate the yeast enzyme. This finding of the Ec enzyme is economically beneficial, suggesting that lower costs are involved to produce high quantities of 3 -amino-tailed tRNA. Other structurally related CCA enzymes may work also, but not archaeal enzymes, which do not catalyze pyrophosphorolysis (Igarashi et al. 2011). For general application of protocol 2, the stably charged amide-linked aa-tRNA can be separated from uncharged tRNA by a brief alkali treatment followed by HPLC through a reverse phase column (Polikanov et al. 2014) or by electrophoresis through a denaturing polyacrylamide gel. Such purified aa-tRNAs are now accessible to a wide range of studies to explore both canonical and noncanonical activities.

\section{MATERIALS AND METHODS}

\section{Isolation of tRNAs and enzymes}

All tRNAs used in this study, Ec tRNA ${ }^{\text {Pro/UGG }}$ (where U34 of the wobble position is modified to $\mathrm{mcmo}^{5} \mathrm{U} 34$ ) (Masuda et al. 2018), tRNA ${ }^{A r g / I C G}$ (where 134 is inosine), tRNA ${ }^{\text {Met/CAU }}$ (the initia-

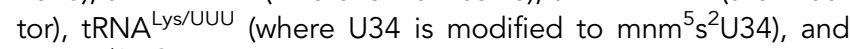
tRNA ${ }^{\mathrm{Val} / \mathrm{UAC}}$ (where U34 is modified to $\mathrm{cmo}^{5} \mathrm{U} 34$ ), were purified from native $E$. coli cells by an affinity purification procedure (Liu et al. 2011). Ec CCA enzyme was purified from a recombinant clone overexpressed in E. coli BL21(DE3) (Dupasquier et al. 2008). The gene for Ec RNase T was cloned into pET22-b and 
the recombinant protein was purified from BL21(DE3). Both proteins were stored in $50 \mathrm{mM}$ Tris- $\mathrm{HCl}, \mathrm{pH} 7.5,1 \mathrm{mM} \mathrm{DTT}$, and $40 \%$ glycerol at $-20^{\circ} \mathrm{C}$. Recombinant aaRS enzymes were purified as previously described (Zhang et al. 2006).

\section{3 '-amino-tailing of tRNA}

Protocol 1. An EctRNA $(17 \mu \mathrm{M})$ and Ec CCA enzyme $(17 \mu \mathrm{M})$ were incubated with nATP $(1 \mathrm{mM})$ in the CCA buffer at $37^{\circ} \mathrm{C}$ for $30 \mathrm{~min}$. The reaction was phenol extracted, ethanol precipitated, and the product tRNA was resuspended in TE.

Protocol 2. An Ec tRNA $(17 \mu \mathrm{M})$ and Ec CCA enzyme $(17 \mu \mathrm{M})$ were incubated with $\mathrm{nATP}(6 \mathrm{mM})$ and PPi $(1 \mathrm{mM})$ in the CCA buffer at $37^{\circ} \mathrm{C}$ for $1 \mathrm{~h}$. The reaction was phenol extracted, ethanol precipitated, and the product tRNA was resuspended in TE.

Protocol 3. An Ec tRNA $(17 \mu \mathrm{M})$ and Ec CCA enzyme $(17 \mu \mathrm{M})$ were incubated with PPi $(0.25 \mathrm{mM})$ in the CCA buffer at $37^{\circ} \mathrm{C}$ for $10 \mathrm{~min}$. The reaction was twofold diluted with CCA buffer containing PPiase (0.4 units), CTP (20 mM), and nATP (4 mM) and incubated at $37^{\circ} \mathrm{C}$ for an additional $30 \mathrm{~min}$. The reaction was phenol extracted, ethanol precipitated, and the product tRNA was resuspended in TE.

Protocol 4. An Ec tRNA $(17 \mu \mathrm{M})$ and Ec CCA enzyme $(17 \mu \mathrm{M})$ were incubated with PPi $(0.25 \mathrm{mM})$ and CTP $(0.62 \mathrm{mM})$ in the CCA buffer at $37^{\circ} \mathrm{C}$ for $10 \mathrm{~min}$. The reaction was twofold diluted with CCA buffer containing PPiase (0.4 units) and nATP ( $4 \mathrm{mM})$ and incubated at $37^{\circ} \mathrm{C}$ for an additional $30 \mathrm{~min}$. The reaction was phenol extracted, ethanol precipitated, and the product tRNA was resuspended in TE.

Protocol 5. An Ec tRNA $(17 \mu \mathrm{M})$ and Ec RNase T $(170 \mu \mathrm{M})$ were incubated in the RNase $\mathrm{T}$ buffer at $37^{\circ} \mathrm{C}$ for $30 \mathrm{~min}$. The reaction was phenol extracted, ethanol precipitated, and the tRNA was resuspended in TE. The tRNA (17 uM) was then reacted with Ec CCA enzyme $(17 \mu \mathrm{M})$ and $\mathrm{nATP}(1 \mathrm{mM})$ in the CCA buffer at $37^{\circ} \mathrm{C}$ for 30 min. The reaction was phenol extracted, ethanol precipitated, and the product tRNA was resuspended in TE.

\section{Biotin-streptavidin conjugation}

Tailed tRNA (7 pmol) was incubated for $1 \mathrm{~h}$ at room temperature in $60 \mathrm{mM}$ Hepes, pH 8.0, with $15 \mu \mathrm{M}$ sulfo-NHS-biotin (Thermo Fisher). Following two consecutive ethanol precipitations, each with a $70 \%$ ethanol wash, the tRNA pellet was dissolved in $4 \mu \mathrm{L}$ water with $1 \mathrm{mg} / \mathrm{mL}$ streptavidin. After $20 \mathrm{~min}$ at room temperature, a $2 \mu \mathrm{L}$ aliquot was diluted twofold with the denaturing loading solution and electrophoresed through an analytical denaturing $12 \% \mathrm{PAGE} / 7 \mathrm{M}$ urea gel run at $200 \mathrm{~V}$ for $50 \mathrm{~min}$. tRNA bands were visualized by staining with SYBR gold. The method was developed from a previous publication (Murakami et al. 2003).

\section{Aminoacylation of tRNA}

Aminoacylation of tRNA was performed using the cognate aaRS and ${ }^{3} \mathrm{H}$-amino acid as previously described (Shitivelband and Hou 2005; Zhang et al. 2006). Charged tRNAs were extracted with $\mathrm{pH} 5.0$ phenol and ethanol precipitated. Charging levels were determined before and after alkali hydrolysis in $0.1 \mathrm{M}$ gly- cine $\mathrm{pH} 9.0$ for $3.5 \mathrm{~h}$ at $37^{\circ} \mathrm{C}$. After alkali hydrolysis, the aatRNA was passed through a gel filtration cartridge (CentriSpin 20; Princeton Separations) in $25 \mathrm{mM} \mathrm{NaOAc}$ buffer ( $\mathrm{pH}$ 5.0), which allowed the aa-tRNA to flow through. The concentration of the tRNA and amino acid of the flow through were determined, respectively, from A260 measurement and liquid scintillation counting.

\section{ACKNOWLEDGMENTS}

We thank Isao Masuda for discussion and presentation of figures and Yury Polikanov for discussion. This work was supported by National Institutes of Health grants GM108972, GM114343, and GM126210 to Y.M.H.

Received July 8, 2018; accepted September 7, 2018.

\section{REFERENCES}

Dare K, Ibba M. 2012. Roles of tRNA in cell wall biosynthesis. Wiley Interdiscip Rev RNA 3: 247-264.

Dupasquier M, Kim S, Halkidis K, Gamper H, Hou YM. 2008. tRNA integrity is a prerequisite for rapid CCA addition: implication for quality control. J Mol Biol 379: 579-588.

Eriani G, Delarue M, Poch O, Gangloff J, Moras D. 1990. Partition of tRNA synthetases into two classes based on mutually exclusive sets of sequence motifs. Nature 347: 203-206.

Francis TA, Ehrenfeld GM, Gregory MR, Hecht SM. 1983. Transfer RNA pyrophosphorolysis with CTP(ATP):tRNA nucleotidyltransferase. A direct route to tRNAs modified at the $3^{\prime}$ terminus. J Biol Chem 258: 4279-4284.

Francklyn C, Perona JJ, Puetz J, Hou YM. 2002. Aminoacyl-tRNA synthetases: versatile players in the changing theater of translation. RNA 8: 1363-1372.

Fraser TH, Rich A. 1973. Synthesis and aminoacylation of 3'-amino-3'deoxy transfer RNA and its activity in ribosomal protein synthesis. Proc Natl Acad Sci 70: 2671-2675.

Gamper HB, Masuda I, Frenkel-Morgenstern M, Hou YM. 2015. Maintenance of protein synthesis reading frame by EF-P and $\mathrm{m}^{1} \mathrm{G} 37$-tRNA. Nat Commun 6: 7226.

Gondry M, Jacques IB, Thai R, Babin M, Canu N, Seguin J, Belin P, Pernodet JL, Moutiez M. 2018. A comprehensive overview of the cyclodipeptide synthase family enriched with the characterization of 32 new enzymes. Front Microbiol 9: 46.

Hansen JL, Schmeing TM, Moore PB, Steitz TA. 2002. Structural insights into peptide bond formation. Proc Natl Acad Sci 99: 11670-11675.

Hou YM. 2000. Unusual synthesis by the Escherichia coli CCA-adding enzyme. RNA 6: 1031-1043.

Hou YM. 2010. CCA addition to tRNA: implications for tRNA quality control. IUBMB Life 62: 251-260.

Hou YM, Gu SQ, Zhou H, Ingerman L. 2005. Metal-ion-dependent catalysis and specificity of CCA-adding enzymes: a comparison of two classes. Biochemistry 44: 12849-12859.

Igarashi T, Liu C, Morinaga H, Kim S, Hou YM. 2011. Pyrophosphorolysis of CCA addition: implication for fidelity. J Mol Bio/ 414: 28-43.

Kashina A. 2014. Protein arginylation, a global biological regulator that targets actin cytoskeleton and the muscle. Anat Rec (Hoboken) 297: 1630-1636.

Kim S, Liu C, Halkidis K, Gamper HB, Hou YM. 2009. Distinct kinetic determinants for the stepwise CCA addition to tRNA. RNA 15: 1827-1836. 
Ledoux S, Uhlenbeck OC. 2008. [3'-32P]-labeling tRNA with nucleotidyltransferase for assaying aminoacylation and peptide bond formation. Methods 44: 74-80.

Liu C, Gamper H, Liu H, Cooperman BS, Hou YM. 2011. Potential for interdependent development of tRNA determinants for aminoacylation and ribosome decoding. Nat Commun 2: 329.

Luer C, Schauer S, Mobius K, Schulze J, Schubert WD, Heinz DW, Jahn D, Moser J. 2005. Complex formation between glutamyltRNA reductase and glutamate-1-semialdehyde 2,1-aminomutase in Escherichia coli during the initial reactions of porphyrin biosynthesis. J Biol Chem 280: 18568-18572.

Masuda I, Takase R, Matsubara R, Paulines MJ, Gamper H, Limbach PA, Hou YM. 2018. Selective terminal methylation of a tRNA wobble base. Nucleic Acids Res 46: e37.

Murakami H, Kourouklis D, Suga H. 2003. Using a solid-phase ribozyme aminoacylation system to reprogram the genetic code. Chem Biol 10: 1077-1084.

Pang YL, Poruri K, Martinis SA. 2014. tRNA synthetase: tRNA aminoacylation and beyond. Wiley Interdiscip Rev RNA 5: 461480.
Peacock JR, Walvoord RR, Chang AY, Kozlowski MC, Gamper H, Hou YM. 2014. Amino acid-dependent stability of the acyl linkage in aminoacyl-tRNA. RNA 20: 758-764.

Polikanov YS, Steitz TA, Innis CA. 2014. A proton wire to couple aminoacyl-tRNA accommodation and peptide-bond formation on the ribosome. Nat Struct Mol Biol 21: 787-793.

Shitivelband S, Hou YM. 2005. Breaking the stereo barrier of amino acid attachment to tRNA by a single nucleotide. J Mol Biol 348: 513-521.

Taiji M, Yokoyama S, Miyazawa T. 1983. Transacylation rates of (aminoacyl)adenosine moiety at the $3^{\prime}$-terminus of aminoacyl transfer ribonucleic acid. Biochemistry 22: 3220-3225.

Yue D, Weiner AM, Maizels N. 1998. The CCA-adding enzyme has a single active site. J Biol Chem 273: 29693-29700.

Zhang CM, Perona JJ, Ryu K, Francklyn C, Hou YM. 2006. Distinct kinetic mechanisms of the two classes of aminoacyl-tRNA synthetases. J Mol Biol 361: 300-311.

Zuo Y, Deutscher MP. 2002. Mechanism of action of RNase T. I. Identification of residues required for catalysis, substrate binding, and dimerization. J Biol Chem 277: 50155-50159. 

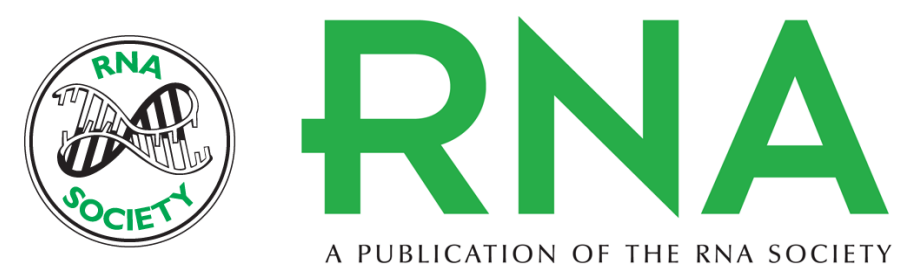

A PUBLICATION OF THE RNA SOCIETY

\section{tRNA 3'-amino-tailing for stable amino acid attachment}

Howard Gamper and Ya-Ming Hou

RNA 2018 24: 1878-1885 originally published online September 14, 2018

Access the most recent version at doi:10.1261/rna.068015.118

References This article cites 28 articles, 10 of which can be accessed free at: http://rnajournal.cshlp.org/content/24/12/1878.full.html\#ref-list-1

Creative This article is distributed exclusively by the RNA Society for the first 12 months after the Commons full-issue publication date (see http://rnajournal.cshlp.org/site/misc/terms.xhtml). After 12 License months, it is available under a Creative Commons License (Attribution-NonCommercial 4.0 International), as described at http://creativecommons.org/licenses/by-nc/4.0/.

Email Alerting Receive free email alerts when new articles cite this article - sign up in the box at the Service top right corner of the article or click here. 